\title{
Marcapasos con sensor de asa cerrada en disautonomía secundaria a enfermedad de Sandhoff
}

\author{
Jorge E. Velásquez ${ }^{\mathrm{a}}$, María C. Gaviriaa ${ }^{\mathrm{a}, \mathrm{b}}$, Valentina L. Estupiñán ${ }^{\mathrm{a}}$, \\ Juan C. Díaz-Martínez ${ }^{a}$, Julián M. Aristizábal ${ }^{a, b}$, Jorge E. Marín ${ }^{a}$, William Uribe ${ }^{a}$ \\ y Mauricio Duque ${ }^{\mathrm{a}, *}$
}

\author{
a Cardiología y Electrofisiología. Universidad CES, CES Cardiología, Medellín, Colombia \\ ${ }^{\mathrm{b}}$ Enlace Cardiología, Medellín, Colombia
}

Recibido el 11 de abril de 2017; aceptado el 19 de junio de 2017

Disponible en Internet el 1 de diciembre de 2017

\author{
PALABRAS CLAVE \\ Marcapasos; \\ Síncope; \\ Sistema nervioso \\ autónomo
}

\begin{abstract}
Resumen
Introducción: en la enfermedad de Sandhoff se ha descrito el compromiso del sistema nervioso autónomo en piel y tracto gastrointestinal; sin embargo, las alteraciones autonómicas del sistema cardiovascular, como la disautonomía, no se han documentado en la literatura revisada. De otro lado, el síncope neuralmente mediado de características cardioinhibitorias, refractario al manejo médico, puede ser tratado con marcapasos con sensor de asa cerrada.

Materiales y métodos: se describe el caso de una paciente de 42 años en quien se realizó el diagnóstico de enfermedad de Sandhof. Asociado a esta patología, presentó disautonomía con episodios de síncope diarios sin respuesta al tratamiento médico, que ameritaron el implante de un marcapasos con sensor de asa cerrada. Posteriormente se revisa la literatura existente sobre el tema.

Conclusiones: hasta la fecha no se había reportado relación entre la enfermedad de Sandhoff y la disautonomía; en el caso de esta paciente el deterioro de la calidad de vida fue significativo. El uso del algoritmo CLS en síncope neuralmente mediado con características cardioinhibitorias, ha mostrado disminuir la cantidad de síncopes en este tipo de pacientes y mejorar la calidad de vida de los mismos.

(c) 2017 Sociedad Colombiana de Cardiología y Cirugía Cardiovascular. Publicado por Elsevier España, S.L.U. Este es un artículo Open Access bajo la licencia CC BY-NC-ND (http:// creativecommons.org/licenses/by-nc-nd/4.0/).
\end{abstract}

\footnotetext{
* Autor para correspondencia.

Correo electrónico: mauricioduquemd@gmail.com (M. Duque).
} 


\section{KEYWORDS}

Pacemakers;

Syncope;

Autonomic nervous

system

\section{Closed-loop pacemakers in dysautonomia secondary to Sandhoff disease}

\begin{abstract}
Introduction: Although involvement of the autonomic nervous system in the skin and gastrointestinal tract has been described in Sandhoff disease, no reports of autonomic changes of the cardiovascular system, such as dysautonomia, have been documented in the literature reviewed. On the other hand, the neurally mediated cardioinhibitory syncope that is refractory to medical management can be treated with pacemakers with closed loop sensors.

Materials and methods: The case is described of a 42 year-old patient who was diagnosed with Sandhoff disease. Associated to this condition, he presented with dysautonomia with daily episodes of syncope, with no response to medical treatment, which warranted the implant of a pacemaker with a closed loop sensor. The existing literature on this topic was subsequently reviewed.

Conclusions: Up until now, there have been no reports on the relationship between Sandhoff disease and dysautonomia. In the case of this patient, the deterioration in the quality of life was significant. The use of the closed loop stimulation (CLS) algorithm in neurally mediated cardioinhibitory syncope has been shown to reduce the number of syncopes in this type of patient and improve their quality of life.

(C) 2017 Sociedad Colombiana de Cardiología y Cirugía Cardiovascular. Published by Elsevier España, S.L.U. This is an open access article under the CC BY-NC-ND license (http:// creativecommons.org/licenses/by-nc-nd/4.0/).
\end{abstract}

\section{Introducción}

La enfermedad de Sandhoff es una condición neurodegenerativa producida por la deficiencia de las hexosaminidasas $A$ y B. El compromiso cardiovascular es poco común, aunque se ha descrito hipertrofia del ventrículo izquierdo, engrosamiento valvular y fusión de las cuerdas tendinosas, además de infiltración de tejido mixoide y fibroso en las valvas y en la íntima de las coronarias. Así mismo, se conoce que causa compromiso del sistema nervioso autónomo, específicamente en piel y tracto gastrointestinal, pero hasta la fecha no se reportan alteraciones en el sistema nervioso autónomo cardíaco.

A continuación, se describe el caso de una paciente de 42 años con enfermedad de Sandhoff, quien posterior al diagnóstico de esta entidad desarrolló episodios de síncope neuralmente mediado de características mixtas, refractario al manejo médico, los cuales pasaron a ser diarios, hasta generar marcado deterioro de la calidad de vida, razón por la cual se decidió implantar marcapasos con sensor de asa cerrada (CLS, su sigla en inglés), evidenciándose mejoría notoria del cuadro clínico.

\section{Caso}

Mujer de 45 años de edad, madre de dos hijos, con antecedentes de fibromialgia, trastorno de ansiedad y depresión, quien consultó por cuadro clínico conformado por fiebre y anemia, por lo que inicialmente se descartó origen infeccioso, deficitario o autoinmune (los principales paraclínicos se reportan en la tabla 1). A pesar de múltiples estudios no se logró esclarecer la causa y por tanto se realizó electroforesis de proteínas y aspirado de médula ósea, ambos interpretados como normales. Los estudios imagenológicos incluyeron colonoscopia, la cual fue normal, y tomografía computarizada (TC) de abdomen que indicó hepatoesplenomegalia. Debido a este hallazgo y ante la persistencia de síntomas y signos, se hizo esplenectomía y biopsia de hígado diagnósticas. La biopsia de bazo mostró hemofagocitos, por lo que Hematología diagnosticó síndrome hemofagocítico y se inició tratamiento con esteroides durante ocho semanas, sin que se lograra la resolución completa del cuadro clínico.

Luego de un año de inicio de los síntomas, se reevaluaron las biopsias de bazo e hígado en otra institución. En la nueva interpretación histopatológica se describió infiltrado de numerosos histiocitos espumosos de citoplasma claro, compatibles con enfermedad de depósito, sin infiltrado linfoide atípico ni granulomas, por lo que se sospechó como primera posibilidad enfermedad de Niemann-Pick del adulto, la cual fue descartada posterior a estudio enzimático negativo para deficiencia de esfingomielinasa. Se continuó, entonces, con la búsqueda de otra enfermedad de depósito, como la enfermedad de Gaucher, que pudiera explicar la persistencia de síntomas y signos. Se midió glucocerebrosidasa en sangre: 5,9 (disminuida) con glucocerebrosidasa en papel de filtro: negativo (pobre aislamiento celular), sin resultado concluyente. Adicionalmente, no se encontraron alteraciones genéticas que apoyaran la sospecha de enfermedad de Gaucher. Fue llevada a una nueva toma de biopsia hepática; los reportes e imágenes se muestran en las figuras 1 a 3 .

Así mismo, se encontraron niveles bajos de hexosaminidasa A ( $56 \%$, rango de normalidad entre 58 y $68 \%$ ), por lo que se llevó a estudio genético que evidenció mutación $\mathrm{S62L}$ en el gen HEXB-subunidad beta de la hexosaminidasa compatible con enfermedad de Sandhoff variante del adulto. Se inició tratamiento con pirimetamina $5 \mathrm{mg} / 24$ horas y se observó mejoría de los síntomas. 
Tabla 1 Exámenes de laboratorio realizados en busca de posible etiología

\begin{tabular}{|c|c|c|}
\hline Paraclínico & Valor & Interpretación \\
\hline Alfa fetoproteína - ng/mL & 3,03 & Normal \\
\hline \multicolumn{3}{|l|}{ Tamizaje reumatológico } \\
\hline Anticuerpos antinucleares - diluciones & Menos de 1:40 & Negativo \\
\hline Anticuerpos anti Smith - UI/ml & 1,2 & Negativo \\
\hline Anticuerpos antii Ro - UI/ml & 1,5 & Negativo \\
\hline Anticuerps anti La - UI/mL & 1,7 & Negativo \\
\hline Anticuerpos anticardiolipinas IgM - UI/mL & 13,3 & Positivo \\
\hline Anticuerpos antiproteinasa $3-\mathrm{UI} / \mathrm{ml}$ & 1,6 & Negativo \\
\hline Anticuerpos antimieloperoxidasa - UI/ml & 2,2 & Negativo \\
\hline Complemento fracción C3 - mg/dl & 147 & Normal \\
\hline Complemento fracción C4 - mg/dl & 25,6 & Normal \\
\hline Factor reumatoide $\mathrm{UI} / \mathrm{mL}$ & 12,7 & Normal \\
\hline \multicolumn{3}{|l|}{ Tamizaje infeccioso } \\
\hline Hepatitis B & - & Negativo \\
\hline ELISA para VIH tipo 1 y 2 & - & Negativo \\
\hline Anticuerpos para Brucella & - & Negativo \\
\hline IgM citomegalovirus & - & Negativo \\
\hline IgM virus Epstein-Barr & - & Negativo \\
\hline
\end{tabular}

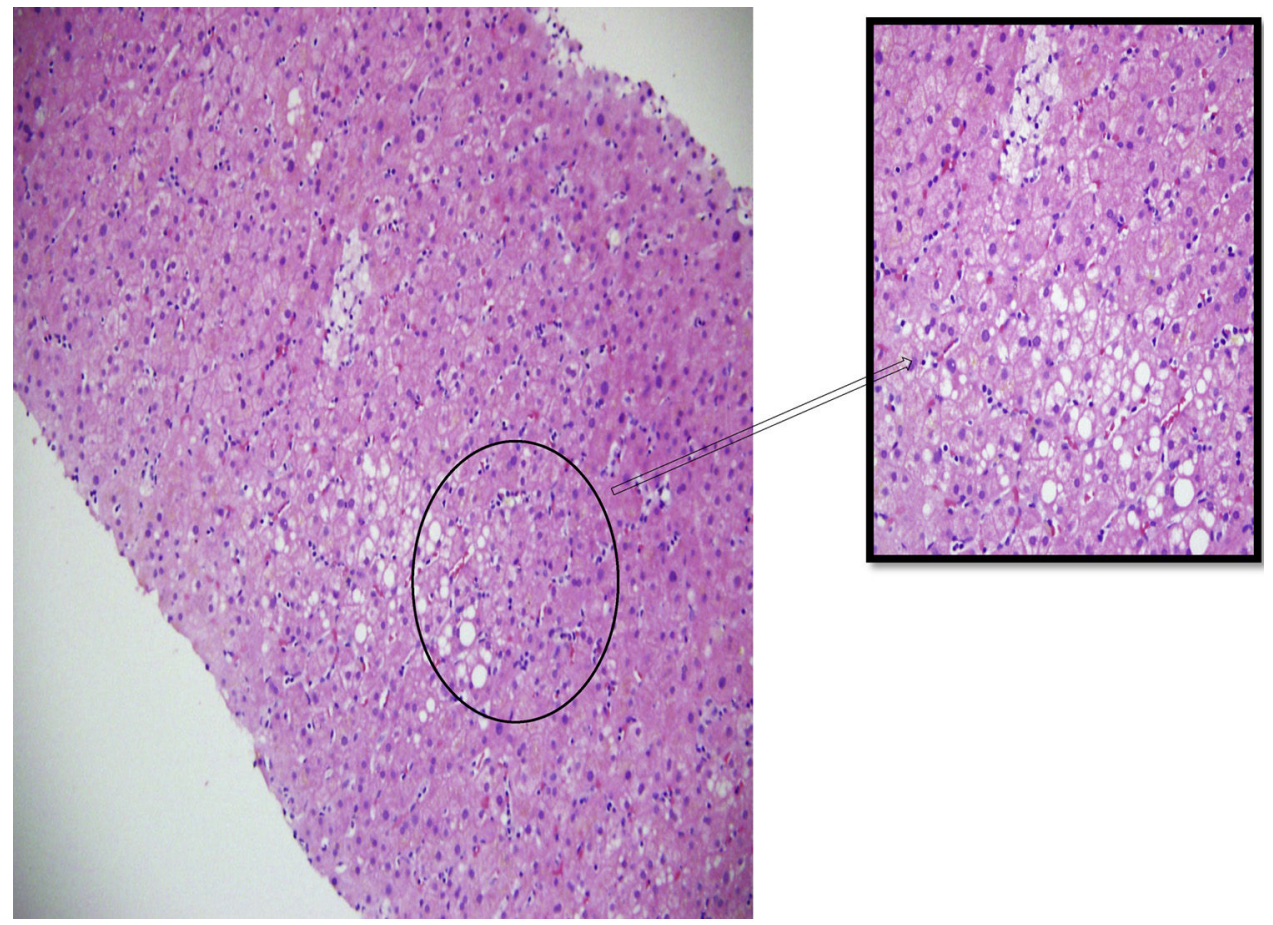

Figura 1 Tinción con hematoxilina y eosina - 200X. Se identifican hepatocitos con esteatosis de tipo mixto con microvacuolas y pigmento de color café en su citoplasma.

Algunos sinusoides ocupados por macrófagos (células de Kupffer) xantomizados (400X) y células inflamatorias circulando en su interior.

Posteriormente, desarrolló episodios de hipotensión, bradicardia sintomática hasta 42 latidos por minuto y síncope. Se descartaron alteraciones neurológicas (RNM cerebral simple y contrastada normal) y estructurales cardiacas (ecocardiografía normal). Se hizo prueba de mesa basculante en la que se documentó síncope neuralmente mediado con respuesta mixta (cardioinhibitoria y vasodepresora). Se inició manejo médico de la disautonomía, el cual consistió en cambios en el estilo de vida, rehabilitación cardíaca y terapia farmacológica con fludrocortisona. Durante los siguientes dos años continuó con episodios de síncope diarios que deterioraron de tal forma su calidad de vida que llegó a imposibilidad de realizar actividades por fuera de su residencia y requerir compañía para asistir a la consulta médica, 


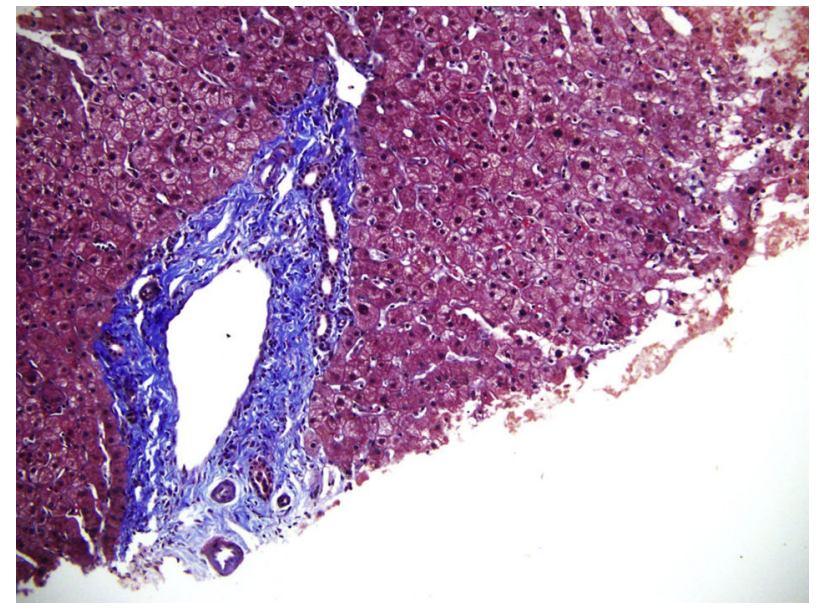

Figura 2 Coloración especial de tricrómico - 200X. En color azul resalta la fibrosis del espacio porta, hallazgo que puede asociarse con enfermedad de Sandhoff.

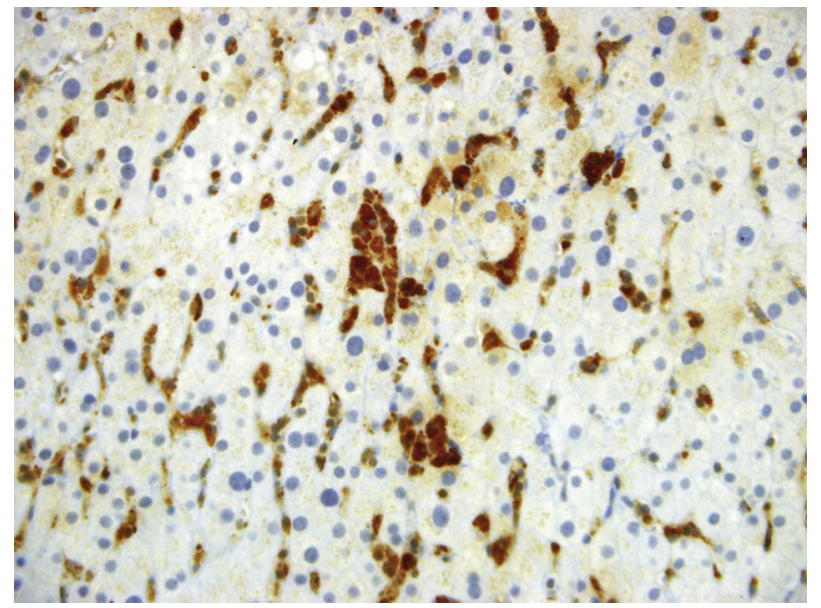

Figura 3 Inmunohistoquímica: CD68 - 400X. Resalta la gran cantidad de células de Kupffer en medio de los sinusoides, algunas de gran tamaño con vacuolas de grasa.

entre otras dificultades. Así mismo, la progresión de su patología continuó y causó mayor deterioro neurológico dado por crisis convulsivas y ataxia, pero con resolución de la hepatomegalia luego del uso de pirimetamina.

En 2015 fue valorada por primera vez por el grupo de CES Cardiología quienes diagnosticaron disfunción sinusal por bradicardia sintomática demostrada en monitorización de 24 horas. Después de un análisis detallado del caso, que incluyó la alteración significativa de la calidad de vida, secundario a los múltiples episodios sincopales refractarios al manejo conservador, se decidió implantar marcapasos con sensor de asa cerrada (CLS, su sigla en inglés) como manejo sintomático. Posterior a esta intervención, presentó un episodio de síncope en los siguientes nueves meses después del implante del dispositivo.

En la actualidad continúa en seguimiento por Electrofisiología; hasta este reporte ya había completado dieciocho meses. Asiste sola a la consulta para reprogramaciones y puede realizar las actividades de la vida normal. Continúa con convulsiones asociadas al deterioro neurológico.

\section{Discusión}

El síncope es una pérdida de conciencia y del tono muscular, con inicio rápido, corta duración y recuperación espontánea completa, secundaria a hipoperfusión cerebral transitoria ${ }^{1}$. Se estima que $50 \%$ de las personas en el mundo tendrán un síncope a lo largo de sus vidas, lo que lo convierte en una patología de alta prevalencia. Tiene dos picos de incidencia, entre los 10-20 años y entre los 60-70 años ${ }^{2}$.

Corresponde al $1 \%$ de las consultas en los servicios de urgencias, de las cuales hasta el $40 \%$ requiere hospitalización, cifra que equivale a 200.000 hospitalizaciones por año en Estados Unidos, con un costo promedio de 5.400 dólares/hospitalización ${ }^{3}$. Afecta de manera significativa la calidad de vida de quienes lo padecen, según lo reporta un estudio publicado en 2006 en el cual se evaluó la calidad de vida durante dos años a pacientes con pérdida transitoria de conciencia y encontró disminución de hasta un $71 \%$ en la capacidad para realizar una rutina diaria, $60 \%$ en la conducción de vehículos, $37 \%$ en la capacidad laboral y $30 \%$ en deterioro de las relaciones interpersonales ${ }^{4}$.

El tipo más frecuente es el vasovagal, presente hasta en el $65 \%$ de los casos. Respecto a la fisiopatología, este tipo se debe a una alteración aguda en las interacciones entre el sistema nervioso autónomo y el cardiovascular, fenómeno que implica una disminución de la perfusión cerebral y en consecuencia, pérdida transitoria de la conciencia; así mismo, se produce una activación inapropiada del reflejo de Bezold-Jarisch por estimulación de los mecanorreceptores ventriculares, secundaria al aumento de presión en el ventrículo izquierdo $y$, por ende, a impendancia ventricular, debida a una contracción más vigorosa que se ocasiona por el llenado ineficiente y no por aumento en el volumen, como sería en activación apropiada. De esta manera, se genera una respuesta paradójica que consiste en vasodepresión (hipotensión) y cardioinhibición (bradicardia) que lleva al síncope ${ }^{1,5,6}$.

En este tipo de síncope el tratamiento inicial es conservador y consiste en cambios en el estilo de vida y control de desencadenantes como el estrés ${ }^{7}$. Sin embargo, la recurrencia es frecuente; se estima que luego de tres episodios de síncope el riesgo es de $36-42 \%^{8}$. Para los casos refractarios al manejo inicial, el tratamiento farmacológico es midodrine, con un grado de recomendación IIb-B $\mathrm{B}^{2,9}$.

En vista de que una cantidad no despreciable de pacientes no responde al tratamiento farmacológico y no farmacológico, se ha utilizado la estimulación cardiaca con marcapasos. Teniendo en cuenta la fisiopatología previamente expuesta, en los pacientes puede predominar la respuesta vasodepresora, cardioinhibitoria o mixta ${ }^{6}$ (hipotensión y bradicardia), como corresponde al caso expuesto. En tal sentido, la terapia de estimulación cardiaca solo sería benéfica para los pacientes con respuesta cardioinhibitoria o mixta.

En el síncope vasovagal y por ortostatismo, la estimulación cardiaca ha sido tema de múltiples estudios, en ocasiones de difícil metodología y reproducibilidad ante lo heterogéneo e impredecible de la enfermedad. En los años noventa comenzaron las primeras investigaciones que compararon la terapia convencional y la estimulación. Pese a encontrar resultados contradictorios, se motivó a extender 
el empleo de dispositivos de estimulación cardiaca para el manejo del síncope con respuesta cardioinhibitoria ${ }^{7}$. Como resultado, posteriormente se llevaron a cabo los tres estudios aleatorizados principales en estimulación cardiaca en síncope: Vasovagal Pacemaker Study ॥ (VPS ॥), Vasovagal Syncope and Pacing Trial (SYNPACE) y Third International Study on Syncope of Unknown Etiology (ISSUE 3) $)^{10-12}$ en los cuales, mediante la estimulación cardiaca con algoritmo de caída de frecuencia cardiaca (DDD-RDR), se demostró disminución en la recurrencia del síncope en un $42 \%$ vs. $33 \%$, $50 \%$ vs. $38 \%$ y $57 \%$ vs. $25 \%$ respectivamente, respecto a la estimulación placebo (ODO); el ISSUE-3 fue el estudio con mayor número de pacientes $(n=511)$.

\section{Algoritmo CLS}

Con base en la fisiopatología de este tipo de síncope, la estimulación con algoritmo de caída de frecuencia cardiaca actúa en etapas tardías del síncope dado que la bradicardia es la última respuesta previa al síncope. Con el uso del algoritmo CLS es posible intervenir de manera precoz, dado que éste mide la impedancia ventricular que refleja la secuencia de contracción cardiaca, y por ende detecta los cambios en la dinámica contráctil, variaciones que llevan a la estimulación de los mecanorreceptores ventriculares previamente descritos y por consiguiente se producen mucho antes que la respuesta vasodepresora y cardioinhibitoria, evitando así la hipotensión y la bradicardia refleja al aumentar la frecuencia cardiaca, lo que, a su vez, mantiene el gasto cardiaco $^{13}$.

En la prueba de mesa basculante se ha demostrado que la intervención temprana del CLS ocurre aproximadamente en 4 minutos, mientras que el síncope aparece 14 minutos más tarde ${ }^{14}$. El primer estudio aleatorizado controlado del CLS fue el INotropy controlled pacing in VAsovagal SYncope (INVASY) publicado en 2004; en este se comparó en un grupo de 50 pacientes la estimulación cardiaca con algoritmo CLS vs. modo DDI con un seguimiento a un año y se demostró disminución en la recurrencia 0 vs. 7 en el grupo control con mejoría en la calidad de vida ${ }^{14}$.

Posteriormente, se llevó a cabo un estudio con un seguimiento más prolongado (36 meses) que confirmaba estos hallazgos con 50 pacientes en dos grupos, uno con estimulación cardiaca algoritmo CLS encendido y otro con éste apagado con diseño cruzado; demostró disminución en los episodios de síncope ( 2 vs. 15$)$ y presíncope (5 vs. 30$)^{15}$. Siguieron más estudios de características similares con igual promedio de pacientes que demostraron el beneficio del algoritmo CLS, entre los cuales se incluyen los de Kanjwal et al. ${ }^{16}$, Palmisano et al. ${ }^{17}$ y Bortnik et al. ${ }^{18}$. En la actualidad se encuentra en proceso de reclutamiento el que será el estudio con mayor número de pacientes (se estiman 128) para la valoración del algoritmo CLS vs. estimulación cardiaca placebo (ODO); la publicación de sus resultados se proyecta para el año $2019^{19}$. Recientemente, se presentó el SPAIN (aún no publicado), que demostró un claro beneficio del sensor CLS en pacientes con síncope neuralmente mediado de características cardioinhibitorias.

\section{Enfermedad de Sandhoff}

Es una condición poco común pero severa, neurodegenerativa, que afecta el almacenamiento lisosomal, y es secundaria a la deficiencia de hexosaminidasas $A$ y $B^{20}$. Su patrón de herencia es autosómico recesivo, con mutación en el gen $H E X B$. Los gangliósidos GM2, que normalmente son catabolizados por las hexosaminidasas, se acumulan en diferentes tejidos como cerebro, hígado, bazo, hueso y corazón ${ }^{21}$, hecho que produce degeneración, autofagia y muerte celular ${ }^{22}$. El compromiso cardiovascular es poco común y está dado por hipertrofia del ventrículo izquierdo, engrosamiento valvular y fusión de las cuerdas tendinosas, además de infiltración de tejido mixoide y fibroso en las valvas y en la íntima de las coronarias ${ }^{23}$. Hasta la fecha poco se ha descrito el compromiso del sistema nervioso autónomo y solo se han reportado siete casos en la literatura, con predominio de compromiso autonómico en piel y tracto gastrointestinal, mas no de compromiso cardiovascular asociado al sistema autónomo ${ }^{24-26}$.

Se conocen tres formas: infantil o clásica, juvenil y del adulto. La más común y más grave es la forma infantil, que inicia entre los 3 y 9 meses de edad; luego de un neurodesarrollo adecuado, el paciente debuta con hipotonía muscular, convulsiones tónico-clónicas, ceguera y retardo en el desarrollo psicomotor ${ }^{20}$. Cuando se desarrolla en niños más grandes se observa espasticidad, ataxia, disartria y pérdida del lenguaje. En adolescentes predominan las alteraciones en el habla, aunadas a incontinencia fecal y urinaria, estas últimas mediadas por disfunción autonómica secundaria a la enfermedad; en la adultez, por su parte, sobresalen los síntomas motores y puede haber o no déficit cognitivo $^{27}$.

Ante la poca prevalencia, el tratamiento es difícil de determinar. A la fecha no existe un tratamiento específico para esta patología y por consiguiente se tratan los síntomas y se indica dieta cetogénica para disminuir el sustrato metabólico de los gangliósidos ${ }^{28}$. En la actualidad se avanza en terapia genómica ${ }^{29,30}$.

En cuanto al caso expuesto, la confirmación de la patología fue difícil y por tanto progresó siguiendo su curso natural; sin embargo, se ha logrado control con el uso de pirimetamina. Adicionalmente, en vista de que el aspecto que más limitaba su calidad de vida eran los episodios de síncope que no mejoraban a pesar de la terapia de primera línea y la convertían en una candidata ideal para la estimulación cardiaca con algoritmo CLS, se logró mejoría sintomática ampliándose así el conocimiento en población colombiana sobre el beneficio de la terapia de estimulación cardiaca con algoritmo CLS.

\section{Conclusiones}

La enfermedad de Sandhoff es una patología neurodegenerativa poco común, la cual se ha asociado con compromiso del sistema autonómo; sin embargo, hasta la fecha no hay reportes que relacionen dicho compromiso con el sistema cardiovascular, especialmente, con disautonomía. En el caso que se reporta, el deterioro de la calidad de vida secundario 
a los síncopes diarios, fue tan significativo que ameritó el implante de un marcapasos con CLS, con lo cual se han obtenido buenos resultados hasta la fecha.

Debe continuarse el estudio y reporte de la enfermedad de Sandhoff y su relación con la disautonomía, así como la evaluación de las ventajas y desventajas de implementar el algoritmo CLS para los síncopes con características neurodegenerativas.

\section{Responsabilidades éticas}

Protección de personas y animales. Los autores declaran que para esta investigación no se han realizado experimentos en seres humanos ni en animales.

Confidencialidad de los datos. Los autores declaran que han seguido los protocolos de su centro de trabajo sobre la publicación de datos de pacientes.

Derecho a la privacidad y consentimiento informado. Los autores declaran que en este artículo no aparecen datos de pacientes.

\section{Financiación}

Ninguna.

\section{Conflicto de intereses}

Ninguno.

\section{Bibliografía}

1. Douglas P. Zipes HGC. Hipotensión y síncope. En: Braunwald Tratado de cardiología. 10 . España: Elsevier; p. 861-71.

2. Developed in collaboration with, European Heart Rhythm Association (EHRA), Heart Failure Association (HFA), and Heart Rhythm Society (HRS), Endorsed by the following societies, European Society of Emergency Medicine (EUSEM), et al. Guidelines for the diagnosis and management of syncope (version 2009): The Task Force for the Diagnosis and Management of Syncope of the European Society of Cardiology (ESC). Eur Heart J. 2009; 30(21):2631-71.

3. Sun BC, Emond JA, Camargo CA. Direct medical costs of syncope-related hospitalizations in the United States. Am J Cardiol. 2005;95:668-71.

4. Van Dijk N, Sprangers MA, Colman N, Boer KR, Wieling W, Linzer M. Clinical factors associated with quality of life in patients with transient loss of consciousness. J Cardiovasc Electrophysiol. 2006;17:998-1003.

5. Brignole M. A new management of syncope: prospective systematic guideline-based evaluation of patients referred urgently to general hospitals. Eur Heart J. 2005;27:76-82.

6. Jhanjee R, Can I, Benditt DG. Syncope. Dis Mon. 2009;55:532-85.

7. Duque $M$, de la OL, Duque M. Tratamiento del síncope neuralmente mediado con marcapasos: utilidad del sensor de asa cerrada. Rev Colomb Cardiol. 2015;22:38-43.

8. Brignole M, Vardas P, Hoffman E, Huikuri H, Moya A, Ricci R, et al., Task Force members. Indications for the use of diagnostic implantable and external ECG loop recorders. Europace. 2009;11:671-87.
9. Izcovich A, Gonzalez Malla C, Manzotti M, Catalano HN, Guyatt G. Midodrine for orthostatic hypotension and recurrent reflex syncope: A systematic review. Neurology. 2014;83: 1170-7.

10. Raviele A, Giada F, Menozzi C, Speca G, Orazi S, Gasparini G, et al. A randomized, double-blind, placebo-controlled study of permanent cardiac pacing for the treatment of recurrent tiltinduced vasovagal syncope. The vasovagal syncope and pacing trial (SYNPACE). Eur Heart J. 2004;25:1741-8.

11. Sutton R, Ungar A, Sgobino P, Russo V, Massa R, Melissano D, et al. Cardiac pacing in patients with neurally mediated syncope and documented asystole: effectiveness analysis from the Third International Study on Syncope of Uncertain Etiology (ISSUE-3) Registry. Europace. 2014;16:595-9.

12. Connolly SJ, Sheldon R, Thorpe KE, Roberts RS, Ellenbogen KA, Wilkoff BL, et al. Pacemaker Therapy for Prevention of Syncope in Patients With Recurrent Severe Vasovagal Syncope: Second Vasovagal Pacemaker Study (VPS II): A Randomized Trial. JAMA. 2003;289:2224.

13. Griesbach L, Schweika O, Huber T, Knote B, Härtel J. Closed loop stimulation: therapy for malignant neurocardiogenic syncope. Prog Biomed Res. 2002;7:242-7.

14. Occhetta E, Bortnik M, Audoglio R, Vassanelli C. Closed loop stimulation in prevention of vasovagal syncope. Inotropy controlled pacing in vasovagal syncope (INVASY): a multicentre randomized, single blind, controlled study. Europace. 2004;6:538-47.

15. Russo V, Rago A, Papa AA, Golino P, Calabrò R, Russo MG, et al. The effect of dual- chamber closed-loop stimulation on syncope recurrence in healthy patients with tilt- induced vasovagal cardioinhibitory syncope: a prospective, randomised, single-blind, crossover study. Heart. 2013;99:1609-13.

16. Kanjwal K, Karabin B, Kanjwal Y, Grubb BP. Preliminary observations on the use of closed-loop cardiac pacing in patients with refractory neurocardiogenic syncope. J Interv Card Electrophysiol. 2010;27:69-73.

17. Palmisano P, Zaccaria M, Luzzi G, Nacci F, Anaclerio M, Favale S. Closed-loop cardiac pacing vs. conventional dual-chamber pacing with specialized sensing and pacing algorithms for syncope prevention in patients with refractory vasovagal syncope: results of a long-term follow-up. Europace. 2012;14:1038-43.

18. Bortnik M, Occhetta E, Dell'Era G, Secco GG, Degiovanni A, Plebani L, et al. Long- term follow-up of DDDR closed-loop cardiac pacing for the prevention of recurrent vasovagal syncope. J Cardiovasc Med. 2012;13:242-5.

19. Benefit of Dual-chamber Pacing With Closed Loop Stimulation (CLS) in Tilt-induced Cardioinhibitory Reflex Syncope - Full Text View - ClinicalTrials.gov [Internet]. [Acceso 27 Dic 2016]. Disponible en: https://clinicaltrials.gov/ct2/show/study/NCT02324920.

20. Saouab R, Mahi M, Abilkacem R, Boumdin H, Chaouir S, Agader O, et al. A Case Report of Sandhoff Disease. Clin Neuroradiol. 2011;21:83-5.

21. Denny CA, Heinecke KA, Kim YP, Baek RC, Loh KS, Butters TD, et al. Restricted ketogenic diet enhances the therapeutic action of $\mathrm{N}$-butyldeoxynojirimycin towards brain GM2 accumulation in adult Sandhoff disease mice: Ketogenic diet, N B-DNJ, and Sandhoff disease. J Neurochem. 2010.

22. Krivit W, Desnick RJ, Lee J, Moller J, Wright F, Sweeley CC, et al. Generalized accumulation of neutral glycosphingolipids with GM2 ganglioside accumulation in the brain. Sandhoff's disease (variant of Tay-Sachs disease). Am J Med. 1972;52:763-70.

23. Guertl B, Noehammer C, Hoefler G. Metabolic cardiomyopathies. Int J Exp Pathol. 2000;81:349-72.

24. Modigliani R, Lemann M, Melançon SB, Mikol J, Potier M, Salmeron $M$, et al. Diarrhea and autonomic dysfunction in a patient with hexosaminidase B deficiency (Sandhoff disease). Gastroenterology. 1994; 106:775-81. 
25. Pellegrini M, Zicari E, Teresa Dotti M, Federico A. Dysautonomic achalasia in two siblings with Sandhoff disease. J Neurol Sci. 2006;241(1-2):107-9.

26. Salman MS, Clarke JT, Midroni G, Waxman MB. Peripheral and autonomic nervous system involvement in chronic GM2gangliosidosis. J Inherit Metab Dis. 2001;24:65-71.

27. Hendriksz CJ, Corry PC, Wraith JE, Besley GTN, Cooper A, Ferrie CD. Juvenile Sandhoff disease-Nine new cases and a review of the literature. J Inherit Metab Dis. 2004;27:241-9.

28. Villamizar-Schiller IT, Pabón LA, Hufnagel SB, Serrano NC, Karl $G$, Jefferies JL, et al. Neurological and cardiac responses after treatment with miglustat and a ketogenic diet in a patient with Sandhoff disease. Eur J Med Genet. 2015;58:180-3.

29. Zhang W, Zeng H, Huang Y, Xie T, Zheng J, Zhao X, et al. Clinical, biochemical and molecular analysis of five Chinese patients with Sandhoff disease. Metab Brain Dis. 2016;31:861-7.

30. Bradbury AM, Peterson TA, Gross AL, Wells SZ, McCurdy VJ, Wolfe KG, et al. AAV-mediated gene delivery attenuates neuroinflammation in feline Sandhoff disease. Neuroscience. 2017;340:117-25. 